Proyecciones

Vol. 21, No 2, pp. 109-125, August 2002.

Universidad Católica del Norte

Antofagasta - Chile

\title{
SPECTRE D'ORDRE UN POUR UN PROBLEME HYPERBOLIQUE ET APPLICATIONS
}

\author{
AOMAR ANANE \\ OMAR CHAKRONE \\ Université Mohamed Premier, Maroc \\ and \\ MOHAMMED GHANIM \\ E. N. C. G., Tanger-Maroc
}

\begin{abstract}
We define and determine the spectrum of order one of the d'Alembertian operator. We resolve the Fredholm alternative and the nonlinear problem at resonance.
\end{abstract}




\section{Introduction}

On se propose de résoudre le problème suivant :

$$
(\mathcal{P})\left\{\begin{array}{lll}
\text { Trouver } & : & u: \bar{\Omega} \times \mathbb{R} \rightarrow \mathbb{R} \text { telle que : } \\
\square u & = & \alpha u+\beta . \nabla u-\gamma u_{t}+g(x, t, u)+h(x, t) \\
& & \text { dans } Q, \\
u(x, t) & = & 0 \text { sur } \partial \Omega \times \mathbb{R}, \\
u(x, t+2 \pi) & = & u(x, t) \text { dans } \Omega \times \mathbb{R}
\end{array}\right.
$$

où $\Omega$ est un domaine borné de $\mathbb{R}^{N}$ de frontière $\left.\partial \Omega, Q=\Omega \times\right] 0,2 \pi[$, $\square u=u_{t t}-\Delta u, h \in L^{2}(Q),(\alpha, \beta, \gamma) \in \mathbb{R} \times \mathbb{R}^{N} \times \mathbb{R}$ et $g: \Omega \times \mathbb{R} \times \mathbb{R} \rightarrow$ $\mathbb{R}$ est une fonction de Carathéodory.

On s'intéresse aux conditions sur $(\alpha, \beta, \gamma)$, $h$ et $g$ pour que le problème $(\mathcal{P})$ admette une solution.

Dans le cas $N=1, \alpha=\gamma=0, \beta=0$ et $h=0$, le problème $(\mathcal{P})$ a été étudié par plusieurs auteurs, citons en particulier [3], [4], [5], [6] , [7] et[8]. Le cas $g(x, t, s)=g(s)$ a été etudié dans [9], le cas $h$ quelconque dans [10], le cas $N=1, \gamma=0, \beta=0$ et $\alpha$ est une valeur propre de $\square u$ a été etudié dans [7] et dans le cas où $\beta=0$ et $\alpha$ est une valeur propre de $\square u+\gamma u_{t}$, le problème $(\mathcal{P})$ a été étudié par Mustonen et Berkovits dans [5], ils ont établi l'existence de solutions avec des conditions de Landesman-Lazer [15] en utilisant un argument d'homotopie.

La situation que nous considérons ici est marquée par la présence d'un terme de transport $\beta . \nabla u$, ce qui constitue une extension du cas étudié par Mustonen et Berkovits dans [5].

L'étude du problème $(\mathcal{P})$ nous a conduit à introduire la notion du spectre d'ordre $1 \sigma_{1}(\square)$ pour le D'Alembertien $\square$, de manière analogue à ce qui a été fait par Anane, Chakrone et Gossez dans [1], [2] et [13] pour le laplacien $\Delta$.

L'introduction du spectre d'ordre un de l'opérateur $\square u$ permet de traiter le problème $(\mathcal{P})$ présentant des dérivées partielles premières au niveau du second membre.

Après avoir déterminé le spectre $\sigma_{1}(\square)$ dans la section 2, nous considérons dans une première étape le cas où $g=0$ et nous établissons (dans la section 3) un résultat de type Alternative de Fredholm "d'ordre 1" en utilisant les propriétés élémentaires de l'opérateur linéaire $\square u-\alpha u-\beta . \nabla u+\gamma u_{t}$. Enfin dans la section 4, nous nous plaçons 
dans une situation de résonance qui correspond au cas où $(\alpha, \beta, \gamma) \in$ $\sigma_{1}(\square)$. Nous montrons, en utilisant des techniques analogues à celles développées dans [5], qu'une condition du type Landesman-Lazer "d'ordre 1" est suffisante pour l'existence de solutions.

\section{Le spectre d'ordre $0 \mathrm{du}$ d'Alembertien :}

Soit $\Omega$ est un domaine borné de $\mathbb{R}^{N}$ de frontière $\partial \Omega$ et $\left.Q=\Omega \times\right] 0,2 \pi[$. Une fonction $u: \mathbb{R} \longrightarrow \mathbb{R}$ est dite $(\theta, 2 \pi)$ - périodique si $u(t+2 \pi)=$ $\theta u(t)$. Pour $\theta \in \mathbb{R}$, nous définissons l'espace de Hilbert $\left(V_{\theta},\|\cdot\|_{\theta}\right)$, par

$$
\begin{gathered}
V_{\theta}=\left\{u: \in L^{2}(Q) ; \quad u_{t}, u_{x_{i}} \in L^{2}(Q), u(\theta, 2 \pi)-\right.\text { périodique et } \\
u(x, t)=0 \text { sur } \partial \Omega \times \mathbb{R}\} .
\end{gathered}
$$

où $\|u\|_{V_{\theta}}^{2}=\|u\|_{L^{2}(Q)}^{2}+\left\|\frac{\partial u}{\partial t}\right\|_{L^{2}(Q)}^{2}+\|\nabla u\|_{L^{2}(Q)}^{2}$. Pour $\theta \neq 0$, on considère le problème aux valeurs propres $\mathcal{P}_{0, \theta}$ suivant

$$
\left(\mathcal{P}_{0, \theta}\right)\left\{\begin{array}{c}
\text { Trouver }(u, \lambda) \in\left(V_{\theta} \backslash\{0\}\right) \times \mathbb{R} \text { tel que : } \\
\square u=\lambda u .
\end{array}\right.
$$

Définissons $\sigma_{o, \theta}(\square)=\left\{\lambda \in \mathbb{R} / \exists u\right.$ avec $(u, \lambda)$ solution de $\left.\mathcal{P}_{0, \theta}\right\}$. On note par $\sigma_{0}(-\Delta)=\left\{\lambda_{i}, \quad i \in \mathbb{N}^{*}\right\}$ le spectre de $-\Delta$ dans $H_{0}^{1}(\Omega)$ (nomé spectre d'ordre 0 ), et par $\left\{\psi_{i}, \quad i \in \mathbb{N}^{*}\right\}$ une base Hilbertienne de $L^{2}(\Omega)$ formée par des fonctions propres associées.

\section{Proposition 1.}

$\sigma_{0, \theta}(\square)=\left\{\begin{array}{clc}\left\{\lambda_{i, k, \theta}, \lambda_{i, \theta} ; \quad i \in \mathbb{N}^{*} \text { et } k \in \mathbb{Z}\right\} & \text { si } & 0<\theta<1, \\ \left\{\lambda_{i, k, \theta} ; \quad i \in \mathbb{N}^{*} \text { et } k \in \mathbb{Z}\right\} & \text { si } & -1 \leq \theta<0 \\ \left\{\lambda_{i, \theta} ; \quad i \in \mathbb{N}^{*} \text { et } k \in \mathbb{Z}\right\} & \text { si } \quad \theta>1, \\ \emptyset & \text { si } \quad \theta<-1\end{array}\right.$

où $\lambda_{i, k, \theta}=\lambda_{i}-\left(\frac{\arccos \theta}{2 \pi}+k\right)^{2}$ et $\lambda_{i, \theta}=\lambda_{i}+\left(\frac{\ln |\theta|}{2 \pi}\right)^{2}$ avec $\phi_{i, k, \theta}=$ $\left.\left.\left[a \cos \left(\left(\frac{\arccos \theta}{2 \pi}+k\right) t\right)\right)+b \sin \left(\frac{\arccos \theta}{2 \pi}+k\right) t\right)\right] \psi_{i}$. (resp. $\left.\phi_{i, \theta}=a e^{\frac{\ln |\theta|}{2 \pi} t} \psi_{i}\right)$. sont les fonctions propres associées à $\lambda_{i, k, \theta}\left(\operatorname{resp} \cdot \lambda_{i, \theta}\right)$. 
Démonstration de la proposition 1. Le problème $\mathcal{P}_{0, \theta}$ peut être résolu par décomposition sur la base Hilbertienne $\left(\psi_{i}\right)_{i}$ de $L^{2}(\Omega)$. Soit $u \in V_{\theta}$, écrivons $u(x, t)=\sum_{i=1}^{+\infty} u_{i}(t) \psi_{i}(x)$. Si $\square u=\lambda u$, alors $\sum_{i=1}^{+\infty}\left[u_{i}^{\prime \prime}(t)+\left(\lambda_{i}-\lambda\right) u_{i}(t)\right] \psi_{i}(x)=0$, donc $u_{i}^{\prime \prime}(t)+\left(\lambda_{i}-\lambda\right) u_{i}(t)=0$ pour tout $i \in \mathbb{N}^{*}$. En écrivant les conditions initiales, nous avons

$$
\left\{\begin{array}{l}
\theta u_{i}(0)=u_{i}(2 \pi) \\
\theta u_{i}^{\prime}(0)=u_{i}^{\prime}(2 \pi) .
\end{array}\right.
$$

Cas1: $\lambda=\lambda_{i}$. Nous avons $u_{i}(t)=c_{i} t+d_{i}$ où

$$
\left\{\begin{aligned}
\theta d_{i} & =2 \pi c_{i}+d_{i} \\
\theta c_{i} & =c_{i} .
\end{aligned}\right.
$$

On vérifie facilement que si $\theta \neq 1$, alors $u_{i}(t)=0$ et si $\theta=1$, alors $u_{i}(t)=d_{i}$ avec $d_{i} \in \mathbb{R}$ quelconque.

Cas2: $\lambda_{i}-\lambda<0$. Nous avons $u_{i}(t)=c_{i} \cosh \left(\omega_{i} t\right)+d_{i} \sinh \left(\omega_{i} t\right)$ où

$$
\left\{\begin{aligned}
\omega_{i}^{2} & =\lambda-\lambda_{i} \\
\theta\left(c_{i}+d_{i}\right) & =\left(c_{i}+d_{i}\right) e^{2 \pi \omega_{i}} \\
\theta\left(c_{i}-d_{i}\right) & =\left(c_{i}-d_{i}\right) e^{-2 \pi \omega_{i}} .
\end{aligned}\right.
$$

On vérifie facilement que si $\theta<0$ ou $\theta=1$, alors $1-\theta e^{2 \pi \omega_{i}} \neq 0$ et $1-\theta e^{-2 \pi \omega_{i}} \neq 0$ donc $c_{i}=d_{i}=0$, par suite $u_{i}(t)=0$. Et si $0<\theta$ avec $\theta \neq 1$, alors $\omega_{i}= \pm \frac{\ln |\theta|}{2 \pi}$, donc $u_{i}(t)=c_{i} e^{\frac{\ln \theta}{2 \pi} t}$ et $\lambda=\lambda_{i}+\left(\frac{\ln \theta}{2 \pi}\right)^{2}$.

Cas3: $\lambda_{i}-\lambda>0$. Nous avons $u_{i}(t)=c_{i} \cos \left(\omega_{i} t\right)+d_{i} \sin \left(\omega_{i} t\right)$, où

$$
\left\{\begin{aligned}
\omega_{i}^{2} & =\lambda_{i}-\lambda, \\
\theta\left(c_{i}^{2}+d_{i}^{2}\right) & =\left(c_{i}^{2}+d_{i}^{2}\right) \cos \left(2 \pi \omega_{i}\right) .
\end{aligned}\right.
$$

On vérifie facilement que si $0<|\theta| \leq 1$, alors $\omega_{i}= \pm \frac{\arccos \theta}{2 \pi}+$ $k, \quad k \in \mathbb{Z}$, donc : $u_{i}(t)=c_{i} \cos \left(\frac{\arccos \theta}{2 \pi}+k\right) t+d_{i} \sin \left(\frac{\arccos \theta}{2 \pi}+k\right) t$, et $\lambda=\lambda_{i}-\left(\frac{\arccos \theta}{2 \pi}+k\right)^{2}$. Et si $|\theta|>1$ alors $u_{i}(t)=0$. Ce qui prouve la proposition.

Corollaire 1. Le spectre d'ordre 0 de $\square$ avec des conditions de périodicité est : $\sigma_{0}(\square)=\sigma_{0,1}(\square)=\left\{\lambda_{i}-k^{2} / \lambda_{i} \in \sigma_{0}(-\Delta), k \in \mathbb{Z}\right\}$. 


\section{Le spectre d'ordre $1 \mathrm{du}$ d'Alembertien}

On considère le problème aux valeurs propres d'ordre 1 suivant :

$\left(\mathcal{P}_{1}\right)\left\{\begin{array}{c}\text { Trouver }(u,(\alpha, \beta, \gamma)) \in\left(V_{1}-\{0\}\right) \times \mathbf{R} \times \mathbb{R}^{N} \times \mathbb{R} \text { tel que : } \\ \square u=\alpha u+\beta . \nabla u-\gamma u_{t} .\end{array}\right.$

Le spectre d'ordre 1 du d'Alembertien $(\square)$ est défini par

$\sigma_{1}(\square)=\left\{(\alpha, \beta, \gamma) ; \quad \exists u \in V_{1} \backslash\{0\}\right.$ avec $(u,(\alpha, \beta, \gamma))$ solution de $\left.\left(\mathcal{P}_{1}\right)\right\}$

Proposition 2. $(u,(\alpha, \beta, \gamma))$ est solution de $\left(\mathcal{P}_{1}\right)$ si et seulement si $\left(s u, \alpha-\frac{\beta^{2}}{4}+\frac{\gamma^{2}}{4}\right)$ est solution de $\left(\mathcal{P}_{0, \theta}\right)$, où $s(x, t)=e^{\frac{\beta}{2} x+\frac{\gamma}{2} t}$ et $\theta=e^{\pi \gamma}$.

Démonstration de la proposition 2. Supposons que $(u,(\alpha, \beta, \gamma))$ est une solution de $\left(\mathcal{P}_{1}\right)$. Posons $v=s u$, il est clair que $v \in V_{\theta} \backslash\{0\}$ et on a succesivement :

$$
\begin{aligned}
& \nabla v=s u \frac{\beta}{2}+s \nabla u \\
& \begin{array}{l}
\Delta v=d i v \nabla v=s \frac{\beta}{2} \cdot \nabla u+u s \frac{(\beta)^{2}}{4}+s \frac{\beta}{2} \cdot \nabla u+s \Delta u=s \beta . \nabla u+u s \frac{\beta^{2}}{4}+s \Delta u ; \\
v_{t}=\frac{\gamma}{2} s u+s u_{t} ; \\
v_{t t}=\frac{\gamma^{2}}{4} s u+\frac{\gamma}{2} s u_{t}+\frac{\gamma}{2} s u_{t}+s u_{t t}=\frac{\gamma^{2}}{4} s u+\gamma s u_{t}+s u_{t t} ; \text { donc } \\
\square v=v_{t t}-\Delta v=\frac{\gamma^{2}}{4} s u+\gamma s u_{t}+s u_{t t}-s \beta \nabla u-u s \frac{(\beta)^{2}}{4}-s \Delta u \\
\quad=\left(\frac{\gamma^{2}}{4}-\frac{\beta^{2}}{4}\right) v+s\left(\square u-\beta \nabla u+\gamma u_{t}\right) \\
\quad=\left(\alpha-\frac{\beta^{2}}{4}+\frac{\gamma^{2}}{4}\right) v .
\end{array}
\end{aligned}
$$

Il en résulte que $\left(s u, \alpha-\frac{\beta^{2}}{4}+\frac{\gamma^{2}}{4}\right)$ est solution du problème $\left(\mathcal{P}_{0, \theta}\right)$, ce qui établit la moitié de la proposition. L'implication réciproque se démontre par un calcul identique.

Corollaire 2. $\sigma_{1}(\square)=\left\{\left(\alpha_{i, k}, \beta, \gamma\right) \in \mathbb{R} \times \mathbb{R}^{N} \times \mathbb{R}^{-}, \quad k, \quad i \in\right.$ $\left.\mathbb{Z} \times \mathbb{N}^{*}\right\} \cup\left\{\left(\alpha_{\mathrm{i}}, \beta, \gamma\right) \in \mathbb{R} \times \mathbb{R}^{\mathrm{N}} \times \mathbb{R}_{*}, i \in \mathbb{Z} \times \mathbb{N}^{*}\right\}$ où $\alpha_{i, k}=\frac{\beta^{2}}{4}-$ $\frac{\gamma^{2}}{4}+\lambda_{i}-\left(\frac{\arccos \left(e^{\pi \gamma}\right)}{2 \pi}+k\right)^{2}$ et $\alpha_{i}=\frac{\beta^{2}}{4}+\lambda_{i}$. 


\section{L'alternative de Fredholm}

Dans toute la suite nous supposerons que l'ensemble $E:=\left\{\lambda_{j}-\right.$ $\left.k^{2}, \quad j \in \mathbb{N}^{*}, \quad k \in \mathbb{Z}\right\}$ est fermé dans $\mathbb{R}$ et que tout point de cet ensemble est isolé (par exemple si $N=1$ et $\Omega=] 0, r \pi[$ avec $r \in \mathbb{Q}$ ).

Étant donnés $(\alpha, \beta, \gamma) \in \mathbb{R} \times \mathbb{R}^{N} \times \mathbb{R}$ et $h \in L^{2}(Q)$. On considère le problème suivant :

$$
\mathcal{P}_{A F, 1}\left\{\begin{array}{l}
\text { trouver } u \in V_{1} \text { tel que } \\
\square u=\alpha u+\beta . \nabla u-\gamma u_{t}+h .
\end{array}\right.
$$

Proposition 3. 1) Si $(\alpha, \beta, \gamma) \in \sigma_{1}(\square)$, alors $\mathcal{P}_{A F, 1}$ admet une solution si et seulement si $\int_{Q} h \phi=0$ pour toute fonction propre $\phi$ associée à $(\alpha,-\beta,-\gamma)$.

2) Si $(\alpha, \beta, \gamma) \notin \sigma_{1}(\square)$, alors $\mathcal{P}_{A F, 1}$ admet une solution pour tout $h \in L^{2}(Q)$.

Lemme 1. (théorème.ii.18 [11] page 29).

Soient $E$ et $F$ deux espaces de Banach et $A: D(A) \subset E \longrightarrow F$ un opérateur linéaire fermé à domaine dense, alors $\left[\operatorname{Ker}\left(A^{*}\right)\right]^{\perp}=\operatorname{Im}(A)$ où $A^{*}$ est l'adjoint de $A$.

\section{Remarques 1.}

a) Pour $(\alpha, \beta, \gamma) \in \mathbb{R} \times \mathbb{R}^{N} \times \mathbb{R}$, on défini l'opérateur

$$
L_{\alpha, \beta, \gamma}: D\left(L_{\alpha, \beta, \gamma}\right) \subset L^{2}(Q) \rightarrow L^{2}(Q): L_{\alpha, \beta, \gamma}(u)=\square u-\alpha u-\beta \nabla u+\gamma u_{t}
$$

où $D\left(L_{\alpha, \beta, \gamma}\right)$ dénote le domaine de l'opérateur $L_{\alpha, \beta, \gamma}$ que nous préciserons par la suite. On vérifie facilement que les points 1) et 2) de la proposition 3 signifient que $\left[\operatorname{Ker}\left(L_{\alpha,-\beta,-\gamma}\right)\right]^{\perp}=\operatorname{Im}\left(L_{\alpha, \beta, \gamma}\right)$.

b) Soit $u \in D\left(L_{\alpha, \beta, \gamma}\right)$, un calcul simple donne

$$
L_{\alpha, \beta, \gamma}(u)=e^{-\frac{\beta}{2} x-\frac{\gamma}{2} t}\left[\square\left(e^{\frac{\beta}{2} x+\frac{\gamma}{2} t} u\right)-\left(\alpha-\frac{\beta^{2}}{4}+\frac{\gamma^{2}}{4}\right)\left(e^{\frac{\beta}{2} x+\frac{\gamma}{2} t} u\right)\right] .
$$

Écrivons $u(x, t)=\sum_{1 \leq i, k \leq+\infty} u_{i, k} \psi_{i}(x) f_{k}(t)$ où $\left(f_{k}\right)_{k}$ est la base Hilbertienne de $L^{2}(] 0,2 \pi[)$ vérifiant $-\left(f_{k}\right)_{t t}=k^{2} f_{k}$ dans $] 0,2 \pi\left[\right.$ et $u_{i, k}=$ 
$\left(u, \psi_{i} f_{k}\right)$ avec $(.,$.$) désigne le produit scalaire de L^{2}(Q)$. On vérifie que

$$
L_{\alpha, \beta, \gamma} u=e^{-\frac{\beta}{2} x-\frac{\gamma}{2} t}\left[\sum_{i, k}\left(\lambda_{i}-k^{2}-\alpha+\frac{\beta^{2}}{4}-\frac{\gamma^{2}}{4}\right)\left(u e^{\frac{\beta}{2} x+\frac{\gamma}{2} t}, \psi_{i} f_{k}\right) \psi_{i} f_{k}\right] .
$$

Donc le domaine et l'image de $L_{\alpha, \beta, \gamma}$ sont définis respectivement par :

$$
\begin{gathered}
D\left(L_{\alpha, \beta, \gamma}\right)=\left\{u \in L^{2}(Q) ; a L a^{6} a N\right. \\
\left.\sum_{i, k}\left(\lambda_{i}-k^{2}-\alpha+\frac{\beta^{2}}{4}-\frac{\gamma^{2}}{4}\right)^{2}\left(u e^{\frac{\beta}{2} x+\frac{\gamma}{2} t}, \psi_{i} f_{k}\right)^{2}<+\infty\right\}, \\
\operatorname{Im}\left(L_{\alpha, \beta, \gamma}\right)=\left\{e^{\frac{-\beta}{2} x-\frac{\gamma}{2} t}\left[\sum_{i, k}\left(\lambda_{i}-k^{2}-\alpha+\frac{\beta^{2}}{4}-\frac{\gamma^{2}}{4}\right)\left(u e^{\frac{\beta}{2} x+\frac{\gamma}{2} t}, \psi_{i} f_{k}\right) \psi_{i} f_{k}\right],\right. \\
\left.u \in D\left(L_{\alpha, \beta, \gamma}\right)\right\} .
\end{gathered}
$$

Par conséquent $D\left(L_{\alpha, \beta, \gamma}\right)$ est dense dans $L^{2}(Q)$ et $\operatorname{Im}\left(L_{\alpha, \beta, \gamma}\right)$ est fermé dans $L^{2}(Q)$.

c) $L_{\alpha, \beta, \gamma}$ est fermé (c'est-à-dire son graphe est fermé). (La démonstration découle de la proposition ii. 16 de [11] page 28).

Démonstration de la proposition 3. D'après le lemme 1 et les remarques 1 (b)et c)), on a

$$
\left[\operatorname{Ker}\left(L_{\alpha, \beta, \gamma}^{*}\right)\right]^{\perp}=\operatorname{Im}\left(L_{\alpha, \beta, \gamma}\right) .
$$

D'autre part, en utilisant la formule de Green, on a pour tout $u, v \in$ $D\left(L_{\alpha, \beta, \gamma}\right)$

$$
\begin{aligned}
\left(L_{\alpha, \beta, \gamma}(u), v\right) & =\int_{Q}(\square u) v-\alpha \int_{Q} u v-\int_{Q}(\beta . \nabla u) v+\gamma \int_{Q} u_{t} v \\
& =\int_{Q} u . \square v-\alpha \int_{Q} u v+\int_{Q} \beta u \nabla v-\gamma \int_{Q} u v_{t} \\
& =\left(u, L_{\alpha,-\beta,-\gamma}(v)\right),
\end{aligned}
$$

d'où $L_{\alpha, \beta, \gamma}^{*}=L_{\alpha,-\beta,-\gamma}$, ce qui prouve la proposition 3 .

\section{Un problème non linéaire à la résonance}

Dans cette section nous étudions l'existence de solutions périodiques du problème

$(\mathcal{P})\left\{\begin{aligned} \square u & =\alpha u+\beta . \nabla u-\gamma u_{t}+g(x, t, u)+h(x, t) \text { dans } Q, \\ u(x, t) & =0 \text { sur } \partial \Omega \times \mathbb{R}, \\ u(x, t+2 \pi) & =u(x, t) \text { dans } \Omega \times \mathbb{R}\end{aligned}\right.$ 
dans le cas de résonance où $(\alpha, \beta, \gamma)$ est une valeur propre d'ordre 1 du d'Alembertien $\left((\alpha, \beta, \gamma) \in \sigma_{1}(\square)\right)$. Avec $h \in \mathrm{E}^{2}(Q)$ et $g: \Omega \times \mathbb{R} \times$ $\mathbb{R} \rightarrow \mathbb{R}$ est une fonction de Carathéodory $2 \pi$-périodique en $t$. Par un mme calcul que dans la démonstration de la proposition 2, on vérifie facilement que $u$ est une solution du problème $(\mathcal{P})$ si et seulement si $v=e^{\frac{\beta}{2} \cdot x} u$ est une solution du problème

$(\tilde{\mathcal{P}})\left\{\begin{aligned} \square v & =\left(\alpha-\beta^{2} / 4\right) v-\gamma v_{t}+\tilde{g}(x, t, v)+\tilde{h}(x, t) \text { dans } Q, \\ v(x, t) & =0 \text { sur } \partial \Omega \times \mathbb{R}, \\ v(x, t+2 \pi) & =v(x, t) \text { dans } \Omega \times \mathbb{R}\end{aligned}\right.$

où $\tilde{g}(x, t, s)=e^{\frac{\beta}{2} \cdot x} g\left(x, t, e^{-\frac{\beta}{2} \cdot x} s\right)$ et $\tilde{h}=e^{\frac{\beta}{2} \cdot x} h$. Posons $\lambda=\alpha-\beta^{2} / 4$, pour tout $u \in D\left(L_{\alpha, \beta, \gamma}\right)$, on note par $T u=\square u+\gamma u_{t}, T_{\lambda}=T-\lambda I$ et $P_{\lambda}: L^{2}(Q) \rightarrow \operatorname{Ker} T_{\lambda}$ la projection orthogonale.

Lemme 2. 1) $T$ est un opérateur linéaire fermé à domaine dense et à image fermé.

2) $T$ est normal (c'est-à-dire il commute avec son adjoint.).

3) $\operatorname{Im}(T)=[\operatorname{Ker}(T)]^{\perp}$.

4) Si $T_{0}$ est la restriction de $T$ sur $\operatorname{Im}(T) \cap D(T)$, alors $T_{0}^{-1}$ : $\operatorname{Im}\left(T_{0}\right) \rightarrow \operatorname{Im}(T) \cap D(T)$ est continu compact.

5) Il existe $c(\alpha, \beta) \in] 0,+\infty]$ tel que pour tout $u \in D(T)$

$$
\left\langle T_{\lambda} u, u\right\rangle \leq \frac{1}{c(\alpha, \beta)}\left\|T_{\lambda} u\right\|^{2}
$$

où

$c(\alpha, \beta)=\min _{\left\{(j, k) \in \mathbb{N}^{*} \times \mathbb{Z} ; \quad \lambda_{\mathrm{j}}-k^{2}>\alpha-\frac{\beta^{2}}{4}\right\}}\left|\lambda_{j}-k^{2}-\alpha+\frac{\beta^{2}}{4}\right|+\frac{\gamma^{2} k^{2}}{\left|\lambda_{j}-k^{2}-\alpha+\frac{\beta^{2}}{4}\right|}$

et $c(\alpha, \beta)=+\infty$ si $\lambda_{j}-k^{2} \leq \alpha-\frac{\beta^{2}}{4}$ avec $c(\alpha, \beta)$ est la valeur optimale vérifiant cette inégalité.

Démonstration du lemme 2. 1) D'après les remarques 1 .

2) Il est clair que $T=T_{\gamma}$ commute avec $T_{\gamma}^{*}=T_{-\gamma)}$.

3) D'après la proposition 1 , on a $\operatorname{Im}(T)=\left[\operatorname{Ker}\left(T^{*}\right)\right]^{\perp}$, et puisque $T$ est normal, on a $\operatorname{Ker} T^{*}=\operatorname{Ker} T$, d'où l'assertion 3 . 
4) $T_{0}$ est bijective de $\operatorname{Im}(T) \cap D(T) \rightarrow \operatorname{Im}\left(T_{0}\right)$ car $\operatorname{Im}(T) \cap$ $\operatorname{Ker}(T)=\{0\}$. Pour la compacité il suffit de composer avec l'injection compacte $H^{1}(Q) \rightarrow L^{2}(Q)$.

5) Soit $L_{C}^{2}(Q)=\left\{u+i v, \quad u, v \in L^{2}(Q)\right\}$ le complexifié de $L^{2}(Q)$. Les lois de $L_{\mathrm{IC}}^{2}(Q)$ sont données par : $x+x^{\prime}:=\left(u+u^{\prime}\right)+i\left(v+v^{\prime}\right)$ et $\lambda x:=(a u-b v)+i(a v+b u)$ pour tout $x=u+i v, x^{\prime}=u^{\prime}+i v^{\prime} \in L_{\mathrm{IC}}^{2}(Q)$ et $\lambda=a+i b \in \mathrm{IC}$. Le produit scalaire de $L_{\mathrm{IC}}^{2}(Q)$ est défini par :

$$
\left\langle x, x^{\prime}\right\rangle_{\mathrm{IC}}=\left\langle u, u^{\prime}\right\rangle+\left\langle v, v^{\prime}\right\rangle+i\left(\left\langle u^{\prime}, v\right\rangle-\left\langle u, v^{\prime}\right\rangle\right) .
$$

D'autre part, nous définissons l'opérateur linéaire complexe $T_{\mathrm{IC}}: D\left(T_{\mathrm{IC}}\right) \subset$ $L_{\mathrm{IC}}^{2}(Q) \rightarrow L_{\mathrm{IC}}^{2}(Q)$ par $D\left(T_{\mathrm{IC}}\right)=\left\{u+i v \in L_{\mathrm{IC}}^{2}(Q), \quad u, v \in D(T)\right\}$ et $T_{\mathrm{IC}}(u+i v)=T u+i T v$ pour tout $u+i v \in D\left(T_{\mathrm{IC}}\right)$. Il est clair, d'après les assertions précédentes, que $\operatorname{Im}\left(T_{\mathrm{IC}}\right)=\left[\operatorname{Ker}\left(T_{\mathrm{IC}}\right)\right]^{\perp}, T_{\mathrm{IC}}$ est normal et que l'inverse de $T_{/ D\left(T_{\mathrm{IC}}\right) \cap \operatorname{Im}\left(T_{\mathrm{IC}}\right)}$ est compact. Par conséquent $T_{\mathrm{IC}}$ admet un spectre dans IC : $\sigma\left(T_{\mathrm{IC}}\right)=\left\{\mu_{j}\right\}_{j \in I}$, où $I \subset \mathbb{Z}$. De plus il existe une base orthonormal $\left\{\phi_{j k} ; \quad j \in I, k=1,2, \ldots, m\left(\mu_{j}\right)\right\}$ de $L_{\mathrm{IC}}^{2}(Q)$ tel que $T_{\mathrm{IC}} \phi_{j k}=\mu_{j} \phi_{j k}, k=1,2, \ldots, m\left(\mu_{j}\right), j \in I$ avec $m\left(\mu_{j}\right)$ est la multiplicité de $\mu_{j}, \mu_{j} \neq 0$ (Notons que $m\left(\mu_{j}\right)<+\infty$ si $\mu_{j} \neq 0$ et $\operatorname{dim} \operatorname{Ker} T_{\mathrm{IC}}$ peut tre infini). D'après [14] (théorème 4, p37) pour tout $w \in D\left(T_{\mathrm{IC}}\right)$, nous avons

$$
T_{\mathrm{IC}} w=\sum_{j \in I} \sum_{k=1}^{m\left(\mu_{j}\right)} \mu_{j}\left\langle w, \phi_{j k}\right\rangle_{\mathrm{IC}} \phi_{j k} .
$$

Remarquons que $T_{\mathrm{IC}}(D(T)) \subset L^{2}(Q)$ et

$$
\begin{gathered}
\langle T u, u\rangle=\left\langle T_{\mathrm{IC}} u, u\right\rangle_{\mathrm{IC}}=\sum_{j, k} R e \mu_{j}\left|\left\langle w, \phi_{j k}\right\rangle_{\mathrm{IC}}\right|^{2}, \\
\left\langle T_{\lambda} u, u\right\rangle=\sum_{j, k}\left(R e \mu_{j}-\lambda\right)\left|\left\langle w, \phi_{j k}\right\rangle_{\mathrm{IC}}\right|^{2}
\end{gathered}
$$

pour tout $u \in D(T)$. D'après (1) et (2), on déduit facilement que

$c(\alpha, \beta)=\inf _{R e \mu_{j}>\lambda} \frac{\left|\mu_{j}-\lambda\right|^{2}}{\operatorname{Re} \mu_{j}-\lambda} \quad \operatorname{avec} c(\alpha, \beta)=+\infty$ si $\operatorname{Re} \mu_{j} \leq \lambda \quad \forall j \in I$. 
Et puisque l'ensemble $E:=\left\{\lambda_{j}-k^{2}, \quad j \in \mathbb{N}^{*}, k \in \mathbb{Z}\right\}$ est fermé dans $\mathbb{R}$ et que tout point de cet ensemble est isolé, on a

$$
\inf _{R e \mu_{j}>\lambda} \frac{\left|\mu_{j}-\lambda\right|^{2}}{R e \mu_{j}-\lambda}=\min _{R e \mu_{j}>\lambda} \frac{\left|\mu_{j}-\lambda\right|^{2}}{R e \mu_{j}-\lambda} \quad \text { avec }
$$

Affirmation. $\sigma\left(T_{C}\right)=\left\{\lambda_{j}-k^{2}+i \gamma k ;(j, k) \in \mathbb{N}^{*} \times \mathbb{Z}\right\}$ avec $\left(i^{2}=\right.$ $-1)$.

Preuve de l'affirmation. Comme $T_{\mathrm{IC}}$ est un opérateur normal complexe, alors $E_{\mu}:=\operatorname{Ker}\left(T_{\mathrm{IC}}-\mu I\right)=\operatorname{Ker}\left(T_{\mathrm{IC}}^{*}-\bar{\mu} I\right)$ pour tout $\mu \in \mathrm{IC}$. Soit $\mu \in \sigma\left(T_{\mathrm{IC}}\right)$ et $u+i v \in E_{\mu} \backslash\{0\}$, on a

$$
\left\{\begin{array}{l}
T_{\mathrm{IC}}(u+i v)=T u+i T v=\mu(u+i v), \\
T_{\mathrm{IC}}^{*}(u+i v)=T^{*} u+i T^{*} v=\bar{\mu}(u+i v) .
\end{array}\right.
$$

Dans notre situation, on a $\square u=(\operatorname{Re} \mu) u, \square v=(\operatorname{Re} \mu) v, \gamma u_{t}=$ $-(\operatorname{Im} \mu) v$ et $\gamma v_{t}=(\operatorname{Im} \mu) u$, puisque $u+i v \neq 0$ (on suppose par exemple $u \neq 0)$, il vient que $\square u=(\operatorname{Re} \mu) u$ et $-\gamma^{2} u_{t t}=(\operatorname{Im} \mu)^{2} u$, d'où $R e \mu \in \sigma_{0}(\square)$ et $\left(\frac{\operatorname{Im} \mu}{\gamma}\right)^{2} \in\left\{k^{2}, \quad k \in \mathbb{Z}\right\}$, d'où l'affirmation 1 , ce qui prouve le lemme 2 .

Nous supposons qu'il existe $\theta>0, a \in L^{2}(Q)$ tel que p.p.x $\in \Omega$, $\forall(t, s) \in] 0,2 \pi[\times \mathbb{R}$, on a

$$
\begin{gathered}
e^{\frac{\beta}{2} \cdot x}\left|g\left(x, t, e^{-\frac{\beta}{2} \cdot x} s\right)\right| \leq \theta|s|+a(x, t), \\
g\left(x, t, e^{-\frac{\beta}{2} \cdot x} s\right) \text { est croissante en } s \quad p . p . x \in \Omega \forall t \in \mathbb{R} .
\end{gathered}
$$

Désignons par $N: L^{2}(Q) \rightarrow L^{2}(Q): N(u)=\tilde{g}(x, t, u)$ l'opérateur de Nemytski associé à $\tilde{g}$.

Lemme 3. . Si g vérifie (5) et (6), alors pour tout $\delta>\theta$ il existe $c_{\delta}(.,$.$) tel que$

$$
\langle N(u)-N(w), u-v\rangle \geq \frac{1}{\delta}\|N(u)\|^{2}-c_{\delta}(w, v)
$$

pour tout $u, v, w \in L^{2}(Q)$. 
Démonstration du lemme 3. Cette démonstration est inspirée du travail de Brézis et Nirenberg dans [12].

Soit $\delta>\theta$, il existe $\varepsilon=\varepsilon(\delta)$ tel que $\frac{1}{\theta}-\frac{\varepsilon}{2}>\frac{1}{\delta}$. pour tout $u, v, w \in$ $L^{2}(Q)$, on a

$\langle N(u)-N(w), u-v\rangle=\langle N(u)-N(w), u-w\rangle+\langle N(u), w-v\rangle-\langle N(w), w-v\rangle$.

D'après (5) et (6), on a

$$
\begin{aligned}
\langle N(u)-N(w), u-w\rangle & =\int_{Q}|\tilde{g}(x, t, u)-\tilde{g}(x, t, w)||u-w| \\
& \geq \int_{Q}|\tilde{g}(x, t, u)-\tilde{g}(x, t, w)|\left\{\frac{|\tilde{g}(x, t, u)|-a(x, t)}{\theta}-|w|\right\} \\
& \geq \frac{1}{\theta}\|N(u)\|^{2}-c(w) N(u)-c(w)
\end{aligned}
$$

où $c(w)$ est une constante positive;

$$
\langle N(u), w-v\rangle \geq-c_{1}(w, v)\|N(u)\| \text { et }\langle N(w), w-v\rangle=c_{2}(w, v)
$$

où $c_{1}$ et $c_{2}$ sont des constantes positives.

En faisant la somme et en tenant compte que $a b \leq \frac{\varepsilon}{2} a^{2}+\frac{1}{2 \varepsilon} b^{2}$ pour tout $a, b \in \mathbb{R}$, il vient

$$
\begin{aligned}
\langle N(u)-N(w), u-v\rangle & \geq \frac{1}{\theta}\|N(u)\|^{2}-c_{3}(w, v) N(u)-c_{4}(w, v) \\
& \geq \frac{1}{\theta}\|N(u)\|^{2}-\frac{\varepsilon}{2}(N(u))^{2}-\frac{2}{2 \varepsilon}\left(c_{3}(w, v)\right)^{2}-c_{4}(w, v) \\
& =\left(\frac{1}{\theta}-\frac{\varepsilon}{2}\right)\|N(u)\|^{2}-c_{\delta}(w, v) \\
& \geq \frac{1}{\delta}\|N(u)\|^{2}-c_{\delta}(w, v) .
\end{aligned}
$$

Ce qui prouve le lemme.

Lemme 4. . [6]. On suppose (5), (6), $\lambda \in \sigma(T)$ et $\lambda \geq 0$. Soit $f \in L^{2}(Q)$, s'il existe $R>0$ tel que

$T(u)-\lambda u-t N(u)-(1-t) P_{\lambda} u \neq t f \quad \forall u \in D(T),\|u\|=R, 0 \leq t \leq 1$,

alors d'équation $T(u)-\lambda u-N(u)=f$ admet une solution $u \in D(T)$ avec $\|u\|<R$.

Démonstration du lemme 4. D'après (5) et (6) $N$ est continue et $\lambda I+N$ est un opérateur monotone, par suite le résultat découle en utilisant l'homotopie étudiée dans [4] voir aussi [3].

Théorème 1. Nous supposons que $g$ vérifie (5) et (6), nous avons 
1) Si $\lambda \geq 0$ (c'est-à-dire $\alpha-\frac{\beta^{2}}{4} \geq 0$ ) et $0<\theta<c(\alpha, \beta)$, alors

$$
\overline{\operatorname{Im} T_{\lambda}-\operatorname{conv} \mathcal{R}(N)}=\overline{\mathcal{R}\left(T_{\lambda}-N\right)}
$$

où $\mathcal{R}(N)=N\left(L^{2}(Q)\right)$ et conv $\mathcal{R}(N)$ désigne l'envelope convexe de $\mathcal{R}(N)$.

2) Si de plus il existe $\eta>0$ et $b \in L^{2}(Q)$ tels que

$$
e^{\frac{\beta}{2} \cdot x}\left|g\left(x, t, e^{-\frac{\beta}{2} \cdot x} s\right)\right| \geq \eta|s|-b(x, t) \quad p \cdot p x \in \Omega, t, s \in \mathbb{R},
$$

alors $\mathcal{R}(N)=L^{2}(Q)$ et $\mathcal{R}\left(T_{\lambda}-N\right)=L^{2}(Q)$ c'est-à-dire le problème $(\mathcal{P})$ admet une solution.

Démonstration du théorème 1. 1) Il suffit de montrer que $\operatorname{Im} T_{\lambda}-\operatorname{conv} \mathcal{R}(N) \subset \overline{\mathcal{R}\left(T_{\lambda}-N\right)}$. Soit $f \in \operatorname{Im} T_{\lambda}-\operatorname{conv} \mathcal{R}(N)$, écrivons $f=T_{\lambda} u_{0}-h$ où $h=\sum_{i=1}^{m} \alpha_{i} N\left(u_{i}\right), \alpha_{i} \geq 0, \sum_{i=1}^{m} \alpha_{i}=1$ et $u_{0} \in D(T)$. Si $f \in \mathcal{R}\left(T_{\lambda}-N\right)$, alors l'assertion est démontrée, sinon l'équation $T_{\lambda} u-N(u)=f$ n'admet pas de solution, ce qui revient à dire que l'équation

$$
T_{\lambda} w-N\left(u_{0}+w\right)+h=0 \quad \text { avec } \quad w=u-u_{0}
$$

n'admet pas de solution. D'après le lemme 4 il existe $\left(w_{n}\right)$ et $\left(t_{n}\right)$ tels que $\left\|w_{n}\right\| \rightarrow+\infty, 0<t_{n}<1$ et

$$
T_{\lambda} w_{n}-\left(1-t_{n}\right) P_{\lambda} w_{n}-t_{n}\left(N\left(u_{0}+w_{n}\right)-h\right)=0 \quad \forall n \in \mathbb{N} .
$$

Comme $T_{\lambda} w_{n}$ et $P_{\lambda} w_{n}$ sont orthogonales, on a

$$
\left\|T_{\lambda} w_{n}\right\| \leq t_{n}\left(\left\|N\left(u_{0}+w_{n}\right)\right\|+\|h\|\right) .
$$

En multipliant (10) par $w_{n}$ et en utilisant le lemme 3 avec $0<\delta<$ $c(\alpha, \beta)$, il vient que

$$
\begin{aligned}
\left\langle T_{\lambda} w_{n}, w_{n}\right\rangle & =\left(1-t_{n}\right)\left\|P_{\lambda} w_{n}\right\|^{2}+t_{n}\left(\left\langle N\left(u_{0}+w_{n}\right), w_{n}\right\rangle-\left\langle h, w_{n}\right\rangle\right) \\
& =\left(1-t_{n}\right)\left\|P_{\lambda} w_{n}\right\|^{2}+t_{n} \sum_{i=1}^{m} \alpha_{i}\left\langle N\left(u_{0}+w_{n}\right)-N\left(u_{i}\right), w_{n}\right\rangle \\
& \geq\left(1-t_{n}\right)\left\|P_{\lambda} w_{n}\right\|^{2}+t_{n}\left\{\frac{1}{\delta}\left\|N\left(u_{0}+w_{n}\right)\right\|^{2}-c(f)\right\} .
\end{aligned}
$$


D'après le lemme 2 assertion 5) et (11), nous avons

$$
\begin{aligned}
\frac{1}{c(\alpha, \beta)} t_{n}^{2}\left(\left\|N\left(u_{0}+w_{n}\right)\right\|+\|h\|\right)^{2} & \geq \frac{1}{c(\alpha, \beta)}\left\|T_{\lambda} w_{n}\right\|^{2} \\
& \geq\left\langle T_{\lambda} w_{n}, w_{n}\right\rangle \\
& \geq \frac{t_{n}^{2}}{\delta}\left\|N\left(u_{0}+w_{n}\right)\right\|^{2}-c(f) \\
& +\left(1-t_{n}\right)\left\|P_{\lambda} w_{n}\right\|^{2} .
\end{aligned}
$$

Comme $\frac{1}{\delta}-\frac{1}{c(\alpha, \beta)}>0$, on déduit que la suite $\left(t_{n}\left\|N\left(u_{0}+w_{n}\right)\right\|\right)_{n}$ est bornée, donc $\left(\left(1-t_{n}\right)\left\|P_{\lambda} w_{n}\right\|^{2}\right)_{n}$ est bornée, et par (11), on a aussi $\left(T_{\lambda} w_{n}\right)_{n}$ est bornée, par suite $\left(Q_{\lambda}\left(w_{n}\right)\right)_{n}:=\left(w_{n}-P_{\lambda} w_{n}\right)_{n}$ est bornée. par conséquent $\left\|P_{\lambda} w_{n}\right\| \rightarrow+\infty$, donc $t_{n} \rightarrow 1$ et $\left(1-t_{n}\right)\left\|P_{\lambda} w_{n}\right\| \rightarrow 0$. Par passage à la limite dans $(10)$, on obtient $T_{\lambda} w_{n}-N\left(u_{0}+w_{n}\right)+h \rightarrow 0$, ce qui prouve que $f \in \overline{\mathcal{R}\left(T_{\lambda}-N\right)}$.

2) D'après (8), on a $\mathcal{R}(N)=L^{2}(Q)$. Soit donc $f \in L^{2}(Q)=$ $\operatorname{Im} T_{\lambda}-\operatorname{conv} \mathcal{R}(N)$, supposons que $f \notin \mathcal{R}\left(T_{\lambda}-N\right)$. Nous procéderons de faon analogue que l'assertion 1 , nous montrons facilement que la suite $\left(N\left(u_{0}+w_{n}\right)\right)_{n}$ est bornée. D'après (8) on déduit que $\left(w_{n}\right)$ est bornée, ce qui est absurde, d'où l'assertion 2 .

Afin d'étudier le problème $(\mathcal{P})$ à la résonance, nous reprenons les notations données dans [12] : Le support de $\mathcal{R}(N)$ est défini par

$$
I_{\mathcal{R}(N)}^{*}(v)=\sup _{u \in L^{2}(Q)}\langle N(u), v\rangle \quad v \in L^{2}(Q)
$$

et la fonction de recession de $N$ est défini par

$$
J_{N}(v)=\liminf _{t \rightarrow+\infty, u \rightarrow v}\langle N(t u), v\rangle \quad v \in L^{2}(Q) .
$$

Notons aussi par

$$
[\tilde{g}, v]=\int_{v>0} \tilde{g}^{+} v+\int_{v<0} \tilde{g}^{-} v, \quad v \in L^{2}(Q),
$$

où

$$
\tilde{g}^{+}(x, t)=\liminf _{s \rightarrow+\infty} \tilde{g}(x, t, s), \quad \tilde{g}^{-}(x, t)=\limsup _{s \rightarrow-\infty} \tilde{g}(x, t, s) .
$$

D'après [12] on a la proposition suivante 
Proposition 4. Soit $h \in L^{2}(Q)$. Si on suppose (5) et (6), alors

1) $[\tilde{g}, v]=J_{N}(v)=I_{\mathcal{R}(N)}^{*}(v)$ pout tout $v \in L^{2}(Q)$.

2) si $\lambda>0$, alors $h$ appartienne à l'interieur de $\operatorname{Im} T_{\lambda}-\operatorname{conv} \mathcal{R}(N)$ si et seulement si $[\tilde{g}, v]>\langle-h, v\rangle$ pour tout $v \in \operatorname{Ker} T_{\lambda} \backslash\{0\}$.

3) si $\lambda=0$, alors $h \in \overline{\operatorname{Im} T_{\lambda}-\operatorname{conv} \mathcal{R}(N)}$ si et seulement si $[\tilde{g}, v] \geq$ $\langle-h, v\rangle$ pour tout $v \in \operatorname{KerT}$.

Maintenant nous sommes en mesure d'énoncer des résultats d'existence pour le problème $(\mathcal{P})$ avec des conditions de résonance de Landesman-Lazer d'ordre 1.

Théorème 2. Soit $h \in L^{2}(Q)$. Si on suppose (5) et (6) avec $0<\theta<$ $c(\alpha, \beta)$, alors.

1) Si $\lambda>0$ et $[\tilde{g}, v]>\langle-h, v\rangle$ pour tout $v \in \operatorname{Ker} T_{\lambda} \backslash\{0\}$, alors le problème $(\mathcal{P})$ admet une solution $u \in D(T)$.

2) Si $\lambda=0$ (c'est-à-dire $\alpha-\frac{\beta^{2}}{4}=0$ ) et $[\tilde{g}, v] \geq\langle-h, v\rangle$ pour tout $v \in \operatorname{Ker} T$, alors le problème $(\mathcal{P})$ admet une solution dans le sens que $h \in \overline{\mathcal{R}(T-N)}$.

Démonstration du théorème 2. 1) D'après la proposition 4, $h \in I m T_{\lambda}-\operatorname{conv} \mathcal{R}(N)$ et d'après le théorème $1, h \in \overline{\mathcal{R}\left(T_{\lambda}-N\right)}$. Donc si $h \in \mathcal{R}(T-N)$, alors l'assertion 1 est démontrée, sinon il existe une suite $\left(u_{n}\right)$ de $D(T)$ tel que

$$
T_{\lambda} u_{n}-N\left(u_{n}\right) \rightarrow f
$$

En utilisant la mme démonstration que le théorème 1, nous montrons facilement que les suites $\left(T_{\lambda} u_{n}\right),\left(N\left(u_{n}\right)\right)$ et $\left(u_{n}-P_{\lambda} u_{n}\right)$ sont bornées et que $\left\|P_{\lambda} u_{n}\right\| \rightarrow+\infty$. Posons $v_{n}=\frac{u_{n}}{\left\|u_{n}\right\|}$, pour une sous suite, nous avons les convergences faibles suivantes $v_{n} \rightarrow v$ et $T_{\lambda} u_{n} \rightarrow \chi \in$ $\operatorname{Im} T_{\lambda}$. Il vient que $P_{\lambda} v_{n} \rightarrow P_{\lambda} v \in \operatorname{Ker} T_{\lambda}$ faiblement et $v_{n}-P_{\lambda} v_{n} \rightarrow$ 0 fortement. Comme $\operatorname{Ker} T_{\lambda}$ est de dimension finie $P_{\lambda} v_{n} \rightarrow P_{\lambda} v$ fortement, donc $v_{n} \rightarrow v=P_{\lambda} v$. D'autre part

$$
\left\langle T_{\lambda} u_{n}, v\right\rangle \rightarrow\langle\chi, v\rangle=0
$$


et par (12), on a $\left\langle N\left(u_{n}\right), v\right\rangle \rightarrow\langle-h, v\rangle$. D'après la proposition 4 , il vient que

$$
\langle-h, v\rangle=\lim _{n \rightarrow+\infty}\left\langle N\left(\left\|u_{n}\right\| v_{n}\right), v\right\rangle \geq J_{N}(v)=[\tilde{g}, v]
$$

ce qui est absurde, d'où l'assertion 1.

L'assertion 2) se démontre facilement en utilisant le théorème 1 et la proposition 4 .

\section{References}

[1] A. Anane-O. Chakrone-J. P. Gossez, Spectre d'ordre supérieure et problème de non résonance, C. R. Acad. Sci.Paris, t325, serie I, pp. 33-36, (1997).

[2] A. Anane-O. Chakrone-J. P. Gossez, Spectre d'ordre supérieure et problème aux limites quasi-linéaires, Bolletino U.M.I. (8) 4-B, pp. 483-519, (2001).

[3] A. K Bennaoum,on the Dirichlet problem for the nonlinear wave equation in bounded domains with corner points, institut de mathématique pure et appliquée université catholique de Louvain-Belgique, recherches de mathematique 51 (1996).

[4] A. K. Bennaoum-J. Mawhin, periodic solutions of some semilinear wave equations on balls and on spheres, institut de Mathématique pure et appliquée université catholique de Louvain-Belgique, recherches de mathématique 15, (1992).

[5] J. Berkovits-V. Mustonen, On the resonance for semilinear equations with normal linear part, J. Math. Maroc 2, pp. 115-123, (1994).

[6] J. Berkovits-V. Mustonen, An extension of Leray-Schauder degree and applications to nonlinear wave equations, Differential and Integral Equations 3, pp. 945-963, (1990). 
[7] J. Berkovits-V. Mustonen, On semilinear wave equations at resonance. proceedings of the first world congress of nonlinear analysts, 1992. Editor: V. Lakshmikantham, Berlin, New York, (1996).

[8] J. Berkovits-V. Mustonen,an application of topologique degree to semilinear equations with nonlinearities strongly monotones, Bull. London Math. Soc. 23, pp. 470-476, (1991).

[9] J. Berkovits-V. Mustonen, On multiple solutions for a class of semilinear wave equations, Nonlinear Analysis: theory and applications, Vol. 16, No. 5, pp. 421-434, (1991).

[10] J. Berkovits-V. Mustonen: On nonresonance for systems of semilinear wave equations, Nonlinear Analysis: theory and applications, Vol. 29, pp. 627-638, (1997)

[11] H. Brézis, Analyse fonctionnelle, théorie et applications, Masson (1987).

[12] H. Brézis-L. Nirenberg, Characterization of the ranges of some nonlinear operators and applications to boundary value problems, Ann. Scuola Norm. Sup. Pisa (4) 5, pp. 225-326, (1978).

[13] O. Chakrone, thèse de doctorat.université d'oujda (1998).

[14] R. Dautray-J. L. Lions, Analyse mathématique et calcul numérique pour les sciences et les techniques, T2 Masson (1985).

[15] E. Landesman, A. Lazer, Nonlinear perturbation of linear elliptic boundary value problems at resonance, J. Math. Mech. 19, pp. $609-623,(1970)$.

Received : December, 2001. 


\section{AOMAR ANANE}

Département de mathématiques

Faculté des Sciences

Université Mohamed Premier

60.000 OUJDA

MAROC

e-mail : anane@sciences.univ-oujda.ac.ma

\section{OMAR CHAKRONE}

Département de mathématiques

Faculté des Sciences

Université Mohamed Premier

60.000 OUJDA

MAROC

e-mail : chakrone@sciences.univ-oujda.ac.ma

\section{MOHAMMED GHANIM}

E. N. C. G.

Tanger - Maroc

e-mail : ghanimmohammed@hotmail.com 